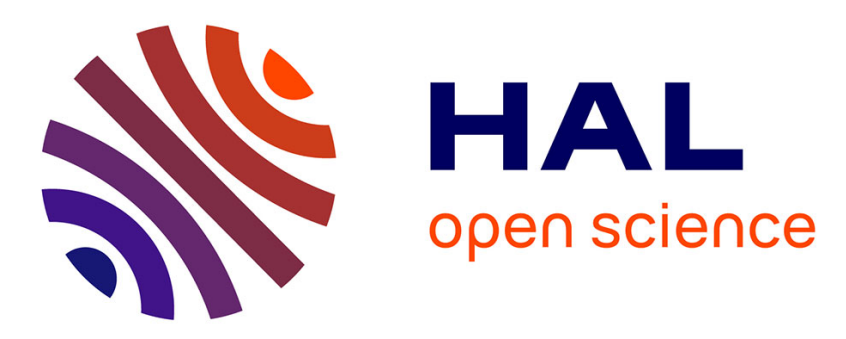

\title{
Une nouvelle estimation extrinsèque du spectre de l'opérateur de Dirac
}

\author{
Nicolas Ginoux
}

\section{To cite this version:}

Nicolas Ginoux. Une nouvelle estimation extrinsèque du spectre de l'opérateur de Dirac. Comptes Rendus. Mathématique, 2003, 336 (10), pp.829-832. 10.1016/S1631-073X(03)00206-1 . hal03117216

\section{HAL Id: hal-03117216 https://hal.science/hal-03117216}

Submitted on 21 Jan 2021

HAL is a multi-disciplinary open access archive for the deposit and dissemination of scientific research documents, whether they are published or not. The documents may come from teaching and research institutions in France or abroad, or from public or private research centers.
L'archive ouverte pluridisciplinaire HAL, est destinée au dépôt et à la diffusion de documents scientifiques de niveau recherche, publiés ou non, émanant des établissements d'enseignement et de recherche français ou étrangers, des laboratoires publics ou privés. 


\title{
Une nouvelle estimation extrinsèque du spectre de l'opérateur de Dirac
}

\author{
Nicolas Ginoux
}

21 janvier 2021

Résumé : Nous établissons une nouvelle majoration optimale pour les plus petites valeurs propres de l'opérateur de Dirac sur une hypersurface compacte de l'espace hyperbolique.

\section{A new extrinsic estimate for the spectrum of the Dirac operator}

\begin{abstract}
We prove a new upper bound for the smallest eigenvalues of the Dirac operator on a compact hypersurface of the hyperbolic space.
\end{abstract}

Soit $\left(M^{m}, g\right)$ une hypersurface riemannienne compacte orientée (de dimension $m$ ) d'une variété riemannienne spinorielle $\left(\widetilde{M}^{m+1}, g\right)$. Notons $\left(\lambda_{k}\right)_{k \geq 1}$ le spectre de l'opérateur de Dirac associé à $g$ et à la structure spinorielle induite sur $M$ (la suite $\left(\left|\lambda_{k}\right|\right)_{k \geq 1}$ étant croissante), et $H$ la courbure moyenne de $M$ dans $\widetilde{M}$. Dans [2], C. Bär montre que, si $N:=2^{\left[\frac{m}{2}\right]}$,

$$
\begin{array}{rlrl}
\lambda_{N}^{2} & \leq \frac{m^{2}}{4 \operatorname{Vol}(M)} \int_{M} H^{2} v_{g}, & & \text { si } \widetilde{M}=\mathbb{R}^{m+1}, \\
\lambda_{N}^{2} & \leq \frac{m^{2}}{4 \operatorname{Vol}(M)} \int_{M}\left(H^{2}+1\right) v_{g}, & & \text { si } \widetilde{M}=S^{m+1}, \\
\left|\lambda_{N}\right| & \leq \frac{m}{2}\left(\sup _{M}|H|+1\right), & \text { si } \widetilde{M}=\mathbb{H}^{m+1} .
\end{array}
$$

L'auteur montre également que, si $M$ est une sphère géodésique, alors (1) et (2) sont des égalités, mais pas (3). Ces résultats appellent donc la question suivante : peut-on améliorer (3) en une majoration optimale?

Nous montrons dans cette note une estimation optimale de $\lambda_{1}$ pour une hypersurface de l'espace hyperbolique. La preuve de ce résultat utilise une approche différente de celle de [9], qui est basée sur les propriétés conformes des opérateurs de Dirac et de Penrose. Nous en discutons ensuite les limites, en remarquant qu'en dimension 2 on ne peut espérer obtenir pour l'opérateur de Dirac une majoration $L^{2}$ analogue à celle de [7] (Théorème 2).

Ce travail, qui repose sur la thèse de l'auteur ([10], Chap. 2), a été effectué à l'Institut Max-Planck pour les Mathématiques dans les Sciences de Leipzig, que l'auteur tient à remercier pour son soutien et son hospitalité.

\section{Préliminaires}

Pour les préliminaires sur la géométrie spinorielle, on se reportera par exemple à [13, 6, 5, 8].

Nous rappelons quelques faits de base sur la restriction de spineurs à une hypersurface (on pourra aussi consulter $[2,14,9])$. Considérons une hypersurface riemannienne compacte (connexe) et orientée $M^{m}$ dans une variété riemannienne spinorielle $\left(\widetilde{M}^{m+1}, g\right)$. L'orientation de $M$ permet, grâce à l'existence sur $M$ d'un champ normal unitaire $\nu$ compatible avec les orientations de $M$ et de $\widetilde{M}$, d'induire une structure spinorielle sur $M$ à partir de celle de $\widetilde{M}$, possédant les propriétés suivantes : notons $\Sigma M(\operatorname{resp} . \Sigma \widetilde{M})$ le 
fibré des spineurs de $(M, g)$ (resp. de $(\widetilde{M}, g)), "<\cdot, \cdot>$ " le produit scalaire hermitien naturel de $\Sigma M, \nabla$ (resp. $\widetilde{\nabla}$ ) la dérivée covariante canonique sur $\Sigma M$ (resp. sur $\Sigma \widetilde{M}$ ), et “" " (resp. ".") la multiplication de Clifford de $T M$ sur $\Sigma M($ resp. de $T \widetilde{M}$ sur $\Sigma \widetilde{M})$.

Il existe alors un isomorphisme

$$
\Sigma \widetilde{M}_{\left.\right|_{M}} \longrightarrow \begin{cases}\Sigma M & \text { si } m \text { est pair } \\ \Sigma M \oplus \Sigma M & \text { si } m \text { est impair }\end{cases}
$$

qui est unitaire et qui envoie, pour tout champ $X$ tangent à $M$ et toute section $\phi$ de $\Sigma \widetilde{M}_{\mid M}$, la section $X \cdot \nu \cdot \phi$ sur $X_{\dot{M}} \phi$ et la section $\widetilde{\nabla}_{X} \phi$ sur $\nabla_{X} \phi+\frac{A(X)}{2} \dot{M}_{M} \phi$, où $A$ est le champ d'endomorphismes de Weingarten de $T M$. L'isomorphisme (4) étant désormais assimilé à l'application identité, considérons l'opérateur $D$ agissant sur les sections de $\Sigma \widetilde{M}_{\left.\right|_{M}}$ par

$$
D:= \begin{cases}D_{M} & \text { si } m \text { est pair } \\ D_{M} \oplus-D_{M} & \text { si } m \text { est impair, }\end{cases}
$$

où $D_{M}$ est l'opérateur de Dirac (dit fondamental) de $(M, g)$. Par définition, $D$ est elliptique autoadjoint et $\operatorname{Spec}\left(D^{2}\right)=\operatorname{Spec}\left(D_{M}^{2}\right)$. Nous noterons, en tenant compte de leurs multiplicités, les valeurs propres de $D_{M}$ par $\lambda_{k}(k \geq 1)$, et supposerons la suite $\left(\left|\lambda_{k}\right|\right)_{k \geq 1}$ croissante.

Afin d'estimer ce spectre en fonction d'invariants extrinsèques, nous comparons $D$ à un opérateur ici auxiliaire appelé opérateur de Dirac-Witten $[15,16]$ et défini dans une base orthonormée locale $\left(e_{j}\right)_{1 \leq j \leq m}$ de $T M$ par $\widehat{D}:=\sum_{j=1}^{m} e_{j} \cdot \widetilde{\nabla}_{e_{j}}$. Grâce aux propriétés de l'isomorphisme (4), les opérateurs $D^{2}$ et $\widehat{D}^{2}$ sont liés par (cf. [10] ou [9], Lemma 2) :

$$
\widehat{D}^{2} \phi=D^{2} \phi-\frac{m}{2} d H_{M} \phi-\frac{m^{2} H^{2}}{4} \phi,
$$

identité valable pour toute section $\phi$ de $\Sigma \widetilde{M}_{\left.\right|_{M}}$.

\section{Résultat principal}

Nous nous restreignons maintenant au cas où la variété ambiante $(\widetilde{M}, g)$ est l'espace hyperbolique réel $\mathbb{H}^{m+1}$ muni de sa métrique standard $g$ à courbure sectionnelle -1 . En tant que variété riemannienne spinorielle, l'espace hyperbolique possède la propriété remarquable d'admettre des spineurs de Killing imaginaires, i.e., il existe des sections non nulles $\phi$ de $\Sigma \widetilde{M}$ satisfaisant, pour tout champ de vecteurs $X$,

$$
\widetilde{\nabla}_{X} \phi= \pm \frac{i}{2} X \cdot \phi
$$

Il est ici à noter que la classification des variétés riemanniennes spinorielles complètes admettant de telles sections a été achevée par H. Baum dans [3] et [4], faisant apparaître d'autres exemples que l'espace hyperbolique. Pour les propriétés des spineurs de Killing, on pourra consulter [5].

Reprenant l'idée introduite dans [2] d'utiliser la restriction de ces spineurs de Killing comme sections-test dans le principe du Min-Max, nous poussons plus loin la preuve du résultat (3) de C. Bär grâce à l'identité (5) qui permet d'éviter l'emploi de l'inégalité de Cauchy-Schwarz (comparer avec [2], p. 586). Nous démontrons le théorème suivant :

Théorème 1 Soit $(M, g)$ une hypersurface riemannienne (immergée) de dimension $m$ compacte et orientée de l'espace hyperbolique $\left(\mathbb{H}^{m+1}, g\right)$. Munissons $M$ de la structure spinorielle induite et posons $N:=2^{\left[\frac{m+2}{2}\right]}$. Alors pour tout $k \in\{1, \ldots, N\}$,

$$
\lambda_{k}^{2} \leq \frac{m^{2}}{4}\left(\sup _{M} H^{2}-1\right) .
$$

De plus, si (7) est une égalité pour $k=1$, alors la courbure moyenne $H$ est constante, et

$$
\lambda_{1}^{2}=\ldots=\lambda_{N}^{2}=\frac{m^{2}}{4}\left(H^{2}-1\right) .
$$


Démonstration : Étant donnée une section non nulle $\phi$ de $\Sigma \mathbb{H}^{m+1}$ satisfaisant (6), évaluons le quotient de Rayleigh $\mathcal{Q}\left(D^{2}, \phi\right):=\frac{\int_{M}<D^{2} \phi, \phi>v_{g}}{\int_{M}<\phi, \phi>v_{g}}$. La section $\phi$ vérifiant (6), il vient immédiatement $\widehat{D} \phi=\mp \frac{m i}{2} \phi$ puis $\widehat{D}^{2} \phi=-\frac{m^{2}}{4} \phi$. D'après (5),

$$
D^{2} \phi=-\frac{m^{2}}{4} \phi+\frac{m^{2} H^{2}}{4} \phi+\frac{m}{2} d H_{M} \phi .
$$

Prenons le produit scalaire hermitien de cette identité avec $\phi$ et identifions-en les parties réelles : puisque la multiplication de Clifford par une 1-forme est anti-autoadjointe (cf. [13]), $\Re \mathrm{e}\left(<d H_{\dot{M}} \phi, \phi>\right)=0$, dont on déduit que :

$$
\Re \mathrm{e}\left(<D^{2} \phi, \phi>\right)=-\frac{m^{2}}{4}<\phi, \phi>+\frac{m^{2} H^{2}}{4}<\phi, \phi>.
$$

Par intégration de cette identité sur $M$, nous obtenons

$$
\mathcal{Q}\left(D^{2}, \phi\right)=-\frac{m^{2}}{4}+\frac{m^{2} \int_{M} H^{2}<\phi, \phi>v_{g}}{4 \int_{M}<\phi, \phi>v_{g}} \leq-\frac{m^{2}}{4}+\frac{m^{2}}{4} \sup _{M} H^{2} .
$$

Puisque l'espace hyperbolique admet un espace de dimension $2.2^{\left[\frac{m+1}{2}\right]}$ de spineurs de Killing imaginaires, l'application du principe du Min-Max donne l'inégalité (7). Remarquons que, si $m$ est impair, le nombre de valeurs propres de $D^{2}$ que l'on peut majorer est $2.2^{\left[\frac{m+1}{2}\right]}$ d'après ce qui précède; cependant, l'opérateur $D^{2}$ est dans ce cas une double copie de $D_{M}^{2}$ d'après le premier paragraphe. C'est pourquoi le nombre de valeurs propres de $D_{M}^{2}$ (ou de $D_{M}$ ) que l'on peut a priori majorer est la moitié de $2.2^{\left[\frac{m+1}{2}\right]}$, i.e., est égal à $N$.

Si maintenant (7) est une égalité pour $k=1$, alors la caractérisation variationnelle de $\lambda_{1}^{2}$ entraine, pour la restriction à $M$ de tout spineur de Killing imaginaire $\phi$ sur $\mathbb{H}^{m+1}$,

$$
D^{2} \phi=\lambda_{1}^{2} \phi
$$

L'injection de cette relation dans (8) et le fait qu'un spineur de Killing imaginaire n'admet pas de zéro [5] donne alors $\lambda_{1}^{2}=\frac{m^{2}}{4}\left(H^{2}-1\right)$, dont on déduit que $H$ doit être constante.

\section{Remarques}

a. Ce résultat se révèle analogue à celui de E. Heintze ([12], Theorem 2.3) pour le laplacien scalaire, et est optimal, car pour toute sphère géodésique de $\mathbb{H}^{m+1},(7)$ est une égalité pour $k=1$ : en effet, pour une sphère géodésique de rayon $r$ (voir [9]),

$$
\frac{m^{2}}{4}\left(H^{2}-1\right)=\frac{m^{2}}{4 \operatorname{sh}^{2}(r)}=\lambda_{1}^{2}=\ldots=\lambda_{N}^{2} .
$$

b. L'inégalité (7) pour $k=1$ demeure évidemment valable sur toute hypersurface (compacte et orientée) de toute variété riemannienne spinorielle admettant un spineur de Killing imaginaire.

c. Pour obtenir un majorant $L^{2}$ analogue à ceux de (1) et (2), il suffirait qu'il existe un spineur de Killing imaginaire de norme constante sur $M$. Il a néanmoins été démontré $[3,4,10]$ que, sous cette hypothèse, $M$ doit être totalement ombilique dans $\mathbb{H}^{m+1}$, i.e., être isométrique à une sphère géodésique de $\mathbb{H}^{m+1}$.

Nous n'excluons pas l'existence lorsque $\widetilde{M}=\mathbb{H}^{m+1}$ d'une telle borne $L^{2}$, qui paraitrait naturelle au regard de l'estimation obtenue par A. El Soufi et S. Ilias ([7], Théorème 1) pour la première valeur propre non nulle du laplacien scalaire. Cependant, nous signalons les limites de cette analogie. On pourrait en effet conjecturer pour l'opérateur de Dirac un résultat semblable à celui démontré par A. El Soufi et S. Ilias pour la plus petite valeur propre strictement positive du laplacien scalaire ([7], Théorème 2) : "si $\widetilde{K}(X, Y)$ désigne la courbure sectionnelle du plan engendré par $X$ et $Y$ tangents à $\widetilde{M}$, et si, dans une base orthonormée locale $\left(e_{j}\right)_{1 \leq j \leq m}$ de $T M$,

$$
R(\iota):=\frac{1}{m(m-1)} \sum_{1 \leq j \neq k \leq m} \widetilde{K}\left(e_{j}, e_{k}\right),
$$

alors la plus petite valeur propre au carré de $D_{M}$ satisfait

$$
\lambda_{1}^{2} \leq \frac{m^{2}}{4 \operatorname{Vol}(M)} \int_{M}\left(H^{2}+R(\iota)\right) v_{g},
$$


et ce pour toute hypersurface compacte d'une quelconque variété $\widetilde{M}$ conformément immergée dans la sphère $S^{m+1}$ ". Or cette conjecture est fausse en dimension $m=2$ : en effet, la quantité $\int_{M}\left(H^{2}+R(\iota)\right) v_{g}$ est dans ce cas la fonctionnelle de Willmore, qui est invariante par changement conforme de métrique sur $\widetilde{M}$. Or le produit $\lambda_{1}^{2} \operatorname{Aire}\left(S^{2}\right)$ est non borné dans la classe conforme de $\operatorname{can}_{S^{2}}$ [1], par conséquent l'inégalité (9) ne peut avoir lieu pour toute métrique de la classe conforme de $\operatorname{can}_{S^{2}}$.

Cette différence de comportement entre l'opérateur de Dirac et le laplacien scalaire se retrouve d'ailleurs dans la caractérisation du cas d'égalité de (2) [11].

Pour l'utilisation des propriétés conformes de l'opérateur de Dirac dans ce contexte, nous renvoyons à $[9,10]$.

\section{Références}

[1] B. Ammann, discussions privées.

[2] C. Bär, Extrinsic Bounds for Eigenvalues of the Dirac Operator, Ann. Glob. Anal. Geom. 16 (1998), $573-596$.

[3] H. Baum, Complete Riemannian manifolds with imaginary Killing spinors, Ann. Glob. Anal. Geom. 7 (1989), 205-226.

[4] _ Odd-dimensional Riemannian manifolds admitting imaginary Killing spinors, Ann. Glob. Anal. Geom. 7 (1989), 141-153.

[5] H. Baum, T. Friedrich, R. Grunewald, I. Kath, Twistor and Killing Spinors on Riemannian Manifolds, Teubner-Texte zur Mathematik 124 (1991), Teubner-Verlag Stuttgart/Leipzig.

[6] J.-P. Bourguignon, O. Hijazi, J.-L. Milhorat, A. Moroianu, A Spinorial approach to Riemannian and Conformal Geometry (en préparation).

[7] A. El Soufi, S. Ilias, Une inégalité de type Reilly pour les sous-variétés de l'espace hyperbolique, Comment. Math. Helv. 67 n 2 (1992), 167-181.

[8] T. Friedrich, Dirac operators in Riemannian geometry, Graduate Studies in Mathematics 25 (2000), American Mathematical Society.

[9] N. Ginoux, Reilly-type spinorial inequalities, Math. Zeit. $241 \mathrm{n}^{0} 3$ (2002), 513-525.

[10] _ Opérateurs de Dirac sur les sous-variétés, Thèse de doctorat (2002), Université Henri Poincaré, Nancy.

[11] _ Remarques sur une estimation de spectre de l'opérateur de Dirac (en préparation).

[12] E. Heintze, Extrinsic upper bound for $\lambda_{1}$, Math. Ann. 280 (1988), 389-402.

[13] H.B. Lawson, M.-L. Michelsohn, Spin Geometry, Princeton University Press (1989).

[14] B. Morel, Eigenvalue Estimates for the Dirac-Schrödinger Operators, J. Geom. Phys. 38 (2001), $1-18$.

[15] E. Witten, A new proof of the positive energy theorem, Commun. Math. Phys. 80 (1981), 381-402.

[16] X. Zhang, Lower bounds for eigenvalues of hypersurface Dirac operators, Math. Res. Lett. 5 (1998), $199-210$.

\section{Nicolas GINOUX}

Max-Planck Institut für Mathematik in den Naturwissenschaften, Inselstraße 22 D-04103 Leipzig. ginoux@mis.mpg.de 